\title{
Opportunistic Relaying in Two-Way Networks
}

\author{
(Invited Paper) \\ Saman Atapattu, Yindi Jing, Hai Jiang, and Chintha Tellambura \\ Department of Electrical and Computer Engineering, University of Alberta, Canada \\ Email: \{atapattu, yindi, hai.jiang, chintha\}@ece.ualberta.ca
}

\begin{abstract}
This paper is on opportunistic relaying for networks with two-way communications. For a network with two users that exchange information with each other through multiple amplifyand-forward relays, we propose a single-relay selection scheme. Since the communication is two-way, the proposed scheme aims at optimizing the worse performance of the two communication tasks between the pair of users. An approximation on the cumulative distribution function (CDF) of the effective worse signal-to-noise ratio (SNR) of the two users using the proposed scheme is obtained, based on which the block error rate (BLER) and the diversity order of the two-way network are derived. Then, the single-relay selection scheme is generalized to a multiplerelay selection scheme with the help of the relay ordering idea. Numerical and simulation results are provided to verify the analysis.
\end{abstract}

Index Terms-Diversity, error rate, relay selection, two-way relay networks.

\section{INTRODUCTION}

Node cooperation has shown to be one of the most effective ways to mitigate the fading effect of wireless channels and achieve spatial diversity in a network. A lot of cooperative strategies have been proposed and their performances have been analyzed (e.g., [1]-[5]). Opportunistic relaying, or relay selection, is one type of cooperation schemes that attracts many interests. In comparison with other schemes, it usually achieves full diversity with less synchronization requirement and overhead. Based on the nature of the relay processing, two popular relaying strategies are decode-and-forward (DF), in which the relays decode the received signals and then forward the decoded information to the destination, and amplify-andforward (AF), in which the relays amplify the received signals and forward them to the destination without a hard decoding [1]- [3]. For AF relaying, a coefficient is used at each relay to constrain its transmit power. Two types of power coefficients are proposed in the literature: the coherent power coefficient and the non-coherent power coefficient, depending on the available channel state information (CSI) at the relay [4], [5]. For one-way relay networks, in which transmission is from one end to the other through relays, relay selection schemes, or opportunistic relaying, such as the best relay selection, the nearest neighbor selection, the best worse channel selection, the maximum harmonic mean selection, etc., are proposed for both DF and AF relaying [6], [7].

Recently, two-way relay networks, or bi-directional relay networks, in which two end users exchange information with each other through a single or multiple common relays, have gained lots of attention. To accomplish the information ex- change between the two end users in a network, the traditional one-way half-duplex scheme requires four channel uses, which is bandwidth inefficient. To improve bandwidth efficiency, a two-way relaying scheme that takes two channel uses only is proposed [8]-[10]. In this scheme, the channels between the source and the relays and between the relays and the destination are active at the same time. Research on relay selection in two-way relay networks, however, is still limited. Some preliminary research work can be found in [11]- [16]. In [11], a relay selection scheme which maximizes the weighted sum-rate capacity in a network with DF relaying is proposed. Based on both instantaneous channel conditions and the queuing status, a cross-layer relay selection metric is investigated in [12]. In [13], the best relay is defined as the relay that maximizes the instantaneous sum-rate function. In [14], a single-relay selection scheme is studied, which minimizes the sum symbol error rate (SER) of both end users. In [15], the relay selection and opportunistic source selection are optimally combined to maximize the mutual information for an AFbased bi-directional relay network. Recently, a single-relay selection scheme that maximizes the worse receive signalto-noise ratio (SNR) of the two end users is proposed for networks with multiple AF relays in [16]. The error rate and diversity order of the network are rigorously analyzed for the high SNR regime.

This paper is on relay selection in two-way networks with multiple AF relays. It is a broader and deeper extension of the work in [16]. We first consider the scenario when only one relay is selected among all available relays, referred to as single-relay selection. For a two-way network with two relays under both the coherent and non-coherent power coefficients, a close approximation on the block error rate (BLER) of the overall network is provided based on an approximation on the cumulative distribution function (CDF) of the worse endto-end SNR. Simulation and analysis show that the coherent power coefficient case always outperforms the non-coherent case. Then, we extend the single-relay selection scheme and analysis to a two-way network with an arbitrary number of relays. From the approximate BLER formula, diversity of the network is derived. After that, we propose a relay ordering function and consider the scenario where multiple relays can be selected, referred to as multiple-relay selection.

The remaining of the paper is organized as follows. The system model, relay selection criterion, and performance metrics are presented in Section II. The single-relay selection is studied in Section III and Section IV for networks with two 
and an arbitrary number of relays, respectively. The multiplerelay selection is considered in Section V. Numerical and simulation results are presented in Section VI, followed by the concluding remarks in Section VII. An involved proof is provided in the appendix.

\section{SySTEM MOdEL}

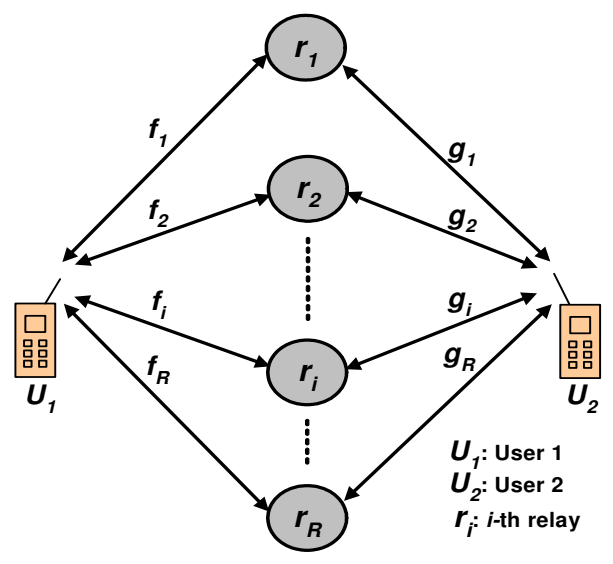

Fig. 1. A two-way multiple-relay network.

\section{A. Network Model and Formulation}

Consider a two-way relay network with two end users (namely, $U_{1}$ and $U_{2}$ ) exchanging information through $R$ relays (namely, $r_{1}, \ldots, r_{R}$ ), as shown in Fig. 1. Each node has a single antenna for both transmission and reception. The fading coefficients between $U_{1}$ and $r_{i}$ and between $U_{2}$ and $r_{i}$ are denoted as $f_{i}$ and $g_{i}$, respectively. All channels are assumed to be independent and identically distributed (i.i.d.) complex Gaussian fading with zero mean and unit variance, i.e., $f_{i}, g_{i} \sim \mathcal{C N}(0,1)$. Therefore, magnitudes of $f_{i}$ and $g_{i}$ follow the Rayleigh distribution. ${ }^{1}$ It is assumed that the two end users know all channel coefficients for decoding. This channel information requirement can be satisfied through training. The CSI requirement at each relay depends on the power coefficient design and will be clarified in Section II-B. The power budget is $P$ for each end user, and $Q_{i}$ for the $i$ th relay.

For simplicity, we assume that both users use the same codebook denoted by $\mathcal{S}$. The information symbols of $U_{1}$ and $U_{2}$ are denoted by $s_{1}$ and $s_{2}$, respectively. They are randomly selected from the codebook. To send $s_{1}$ from $U_{1}$ to $U_{2}$ and also send $s_{2}$ from $U_{2}$ to $U_{1}$, we can use the following twophase protocol. In the first phase, $U_{1}$ and $U_{2}$ transmit $s_{1}$ and $s_{2}$, respectively, at the same time to all relays. Therefore, $r_{i}$ receives the superposition of the two signals, given as

$$
y_{i}=\sqrt{P} f_{i} s_{1}+\sqrt{P} g_{i} s_{2}+v_{i} .
$$

\footnotetext{
${ }^{1}$ Although the diversity and error rate analysis in this paper are for i.i.d Rayleigh flat-fading channels only, the proposed relay selection schemes work for networks with any channel statistics, because the instantaneous CSI is assumed to be available at corresponding nodes.
}

The additive noise at the relays, $v_{i}$ 's, are assumed to be i.i.d. circularly symmetric complex Gaussian random variables with zero mean and unit variance. In the second phase, one of the relays, say the $j$ th relay $r_{j}$, is chosen, based on some relay selection strategy, to transmit (multiple-relay selection is to be discussed in Section V). With AF relaying, $r_{j}$ simply amplifies $y_{j}$, and then forwards the signal to both users. If power coefficient $\alpha_{j}$ is used, the received signals at $U_{1}$ and $U_{2}$ are

$$
\begin{aligned}
& y_{u_{1}, j}=\alpha_{j} f_{j}\left(\sqrt{P} f_{j} s_{1}+\sqrt{P} g_{j} s_{2}+v_{j}\right)+w_{1} \\
& y_{u_{2}, j}=\alpha_{j} g_{j}\left(\sqrt{P} f_{j} s_{1}+\sqrt{P} g_{j} s_{2}+v_{j}\right)+w_{2},
\end{aligned}
$$

respectively. $w_{1}$ and $w_{2}$ denote the noises at the two users, and are assumed to be i.i.d. Gaussian with the distribution $\mathcal{C N}(0,1)$. The equations show that each end user receives an observation that is a combination of the other user's symbol and its own symbol. After canceling the self-interference, $U_{1}$ and $U_{2}$ get

$$
\begin{aligned}
& \tilde{y}_{u_{1}, j}=\alpha_{j} \sqrt{P} f_{j} g_{j} s_{2}+\alpha_{j} f_{j} v_{j}+w_{1} \\
& \tilde{y}_{u_{2}, j}=\alpha_{j} \sqrt{P} f_{j} g_{j} s_{1}+\alpha_{j} g_{j} v_{j}+w_{2},
\end{aligned}
$$

respectively. The users use the following maximum-likelihood (ML) decoding rules to obtain estimations of the other user's symbol:

$$
\begin{aligned}
& \hat{s}_{2}=\arg \min _{s \in \mathcal{S}}\left|\tilde{y}_{u_{1}, j}-\alpha_{j} \sqrt{P} f_{j} g_{j} s\right| \\
& \hat{s}_{1}=\arg \min _{s \in \mathcal{S}}\left|\tilde{y}_{u_{2}, j}-\alpha_{j} \sqrt{P} f_{j} g_{j} s\right| .
\end{aligned}
$$

\section{B. Relay Power Coefficient}

In AF relaying, two types of relay power coefficients have been introduced for one-way relay networks: non-coherent power coefficient, and coherent power coefficient [17], [18]. Similarly, we can use the following two power coefficients at the $j$ th relay in a two-way relay network.

- Non-coherent power coefficient: Relay $j$ has knowledge of the average fading powers of its channels with each user, i.e., $\mathbb{E}\left[\left|f_{j}\right|^{2}\right]$ and $\mathbb{E}\left[\left|g_{j}\right|^{2}\right]$, and uses it to constrain its average transmit power. Therefore, $\alpha_{j}$ is given as

$$
\alpha_{j}^{2}=\frac{Q_{j}}{1+P \mathbb{E}\left[\left|g_{j}\right|^{2}\right]+P \mathbb{E}\left[\left|f_{j}\right|^{2}\right]}=\frac{Q_{j}}{1+2 P} .
$$

Note that $\mathbb{E}[\cdot]$ denotes the expectation, and $|\vartheta|$ denotes the magnitude of a complex value $\vartheta$.

- Coherent power coefficient: Relay $j$ has knowledge of its instantaneous CSI with each user, i.e., $f_{j}$ and $g_{j}$, and uses it to constrain its average transmit power. Therefore, $\alpha_{j}$ is given as

$$
\alpha_{j}^{2}=\frac{Q_{j}}{1+P\left|f_{j}\right|^{2}+P\left|g_{j}\right|^{2}} .
$$

Note that to apply the coherent power coefficient, $r_{j}$ only needs to know the magnitude of $f_{j}$ and $g_{j}$. This is the CSI requirement at $r_{j}$ for single-relay selection. For multiple-relay 
selection, however, the channel phases are also required at the relay for the phase adjustment. The details are given in Section $\mathrm{V}$.

An advantage of the non-coherent power coefficient over the coherent one is in its less overhead. It needs only the knowledge of the average fading powers, while the later needs the instantaneous CSI, which requires training and channel estimation at the relay. But for one-way relay networks, it has been shown that the coherent power coefficient has a better performance. Both power coefficients are considered in our research.

\section{Relay Selection Criterion and Performance Measure}

For one-way networks, the choice of the design criterion is straightforward. If reliability is of interest, usually, the goal is to minimize the error rate; or maximize the end-to-end SNR. For two-way communications, however, there are two communication tasks each with its own bit/symbol error rate and end-to-end SNR. The criterion is less straightforward. The choice depends on the overall network quality-of-service requirement and the fairness consideration. Maximizations of the sum and average error rates of two communication tasks are considered in [14]. In this paper, we care about the reliability of both users and propose to maximize the worse of the end-to-end SNRs of the two users, or equivalent, the larger of the error rates of the two communication tasks.

With single-relay selection, if $r_{j}$ is chosen, the end-to-end SNRs of the two users are given as

$$
\begin{aligned}
U_{1}: \quad \gamma_{u_{1}, j} & =\frac{P Q_{j}\left|f_{j} g_{j}\right|^{2}}{1+2 P+Q_{j}\left|f_{j}\right|^{2}} \\
U_{2}: & \gamma_{u_{2}, j}=\frac{P Q_{j}\left|f_{j} g_{j}\right|^{2}}{1+2 P+Q_{j}\left|g_{j}\right|^{2}},
\end{aligned}
$$

for the non-coherent power coefficient case, and

$$
\begin{aligned}
& U_{1}: \quad \gamma_{u_{1}, j}=\frac{P Q_{j}\left|f_{j} g_{j}\right|^{2}}{1+\left(P+Q_{j}\right)\left|f_{j}\right|^{2}+P\left|g_{j}\right|^{2}} \\
& U_{2}: \quad \gamma_{u_{2}, j}=\frac{P Q_{j}\left|f_{j} g_{j}\right|^{2}}{1+P\left|f_{j}\right|^{2}+\left(P+Q_{j}\right)\left|g_{j}\right|^{2}},
\end{aligned}
$$

for the coherent power coefficient case. In the second-phase of the network communication, we choose Relay $\hat{i}$, i.e., $r_{\hat{i}}$, if it results in the maximum worse end-to-end SNR, i.e.,

$$
\hat{i}=\arg \max _{j} \min \left\{\gamma_{u_{1}, j}, \gamma_{u_{2}, j}\right\} .
$$

As to the performance measure, we consider the block error rate (BLER) and diversity order of the whole network. In a two-way relay network, the two users exchange their symbols, $s_{1}$ and $s_{2}$, with each other. We can take $s_{1}$ and $s_{2}$ as a block $\left(s_{1}, s_{2}\right)$, and say that a block error occurs when either of the two users makes an error, i.e., $\left(\hat{s_{1}}, \hat{s_{2}}\right) \neq\left(s_{1}, s_{2}\right)$. Thus, the BLER metric is an upper bound on the SER of either user, and also a lower bound on the sum of the SERs of the two users. In addition, in the high SNR regime, the user with the worse SNR has a higher probability of making an error, and is the dominant reason of the block error. Thus, the metric is consistent with the relay selection idea and criterion in (8). Later we will show that we can use the worse SNR analysis to approximate the BLER of the two-way network. The details are given in Section III.

Diversity order shows how fast the error rate decreases with the increase in the transmit power. It is conventionally defined as

$$
d=-\lim _{P \rightarrow \infty} \frac{\log \text { Error rate }}{\log \text { Power }}
$$

Usually, the average error rate of a system can be approximated as

$$
\text { Error rate } \approx c P^{-d}
$$

where $c$ relates to the array or coding gain, $P$ is the transmit power, and $d$ is the diversity order [19].

\section{Single-Relay SELECTION IN A TWO-RELAY NETWORK}

In this section, we consider a two-way network with two relays and analyze the performance of the proposed singlerelay selection scheme for the two-way communication. Define $\gamma_{j} \triangleq \min \left\{\gamma_{u_{1}, j}, \gamma_{u_{2}, j}\right\}$. Under the relay selection scheme given in (8), the worse end-to-end SNR can be written as $\gamma_{\hat{i}}=\max _{j} \gamma_{j}$, and $r_{\hat{i}}$ is chosen to cooperate. Since all channels are independent, if the CDF of $\gamma_{j}$ is $F_{\gamma_{j}}(x)$, the CDF of $\gamma_{\hat{i}}$, denoted as $F_{\gamma_{\hat{i}}}(x)$, is $F_{\gamma_{\hat{i}}}(x)=F_{\gamma_{1}}(x) F_{\gamma_{2}}(x)$.

We will derive an average BLER approximation for the twoway network through the analysis on the CDF of the worse end-to-end SNR. For a given channel realization, let $\hat{u}$ be the index of user with the worse receive SNR given that $r_{\hat{i}}$ is selected, i.e., $\hat{u}=1$ if $\gamma_{u_{1}, \hat{i}}<\gamma_{u_{2}, \hat{i}}$ and $\hat{u}=2$ otherwise. Let $\tilde{u}$ be the index of the other user. The BLER can be calculated as follows.

$$
P_{\text {block }}=\mathbb{E}\left[\mathbb{P}\left(\hat{s}_{\tilde{u}} \neq s_{\tilde{u}}\right)\right]+\mathbb{E}\left[\mathbb{P}\left(\hat{s}_{\hat{u}} \neq s_{\hat{u}} \mid \hat{s}_{\tilde{u}}=s_{\tilde{u}}\right)\right] .
$$

$\mathbb{P}$ indicates the probability. The first term is the average probability that the user with the lower SNR makes an error. The second term is the average probability that the user with the higher SNR makes an error given that the other user decodes correctly. When the transmit power is high, the second term is expected to be much smaller. Thus, we approximate the BLER as

$$
P_{\text {block }} \approx P_{\text {appro }}=\mathbb{E}\left[\mathbb{P}\left(\hat{s}_{\tilde{u}} \neq s_{\tilde{u}}\right)\right] .
$$

Since the second term in (10) is non-negative, $P_{\text {appro }}$ actually provides a lower bound on the BLER of the network. From the definition, $P_{\text {appro }}$ is exactly the average SER of a singlesource communication system with receive SNR $\gamma_{\hat{i}}$. With digital modulations, the SER can be approximated as (the nearest neighbor approximation)

$$
P_{\text {appro }} \approx M_{d_{\min }} \mathbb{E}\left[\mathbb{Q}\left(\frac{\beta}{\sqrt{2}} \sqrt{\gamma_{\hat{i}}}\right)\right],
$$

where $M_{d_{\min }}$ is the number of neighbors a constellation point has at the minimum distance $d_{\min }, \beta$ is a constant depending on the modulation, and $\mathbb{Q}(x)$ is the probability that a standard 


$$
\begin{gathered}
I_{1}=\frac{\sqrt{\pi}\left(3 \rho^{2}+12 \rho \eta^{2}+4 \eta^{4}\right) e^{\frac{\eta^{2}}{\rho}} \operatorname{Ercc}\left(\frac{\eta}{\sqrt{\rho}}\right)-2 \sqrt{\rho} \eta\left(5 \rho+2 \eta^{2}\right)}{4 P^{2} \rho^{\frac{9}{2}}}, I_{2}=\frac{\sqrt{\pi}\left(2\left(\rho-\frac{1}{P}\right)+\eta^{2}\right) e^{\frac{\eta^{2}}{4\left(\rho-\frac{1}{P}\right)}} \operatorname{Ercc}\left(\frac{\eta}{2 \sqrt{\left(\rho-\frac{1}{P}\right)}}\right)-2 \eta \sqrt{\rho-\frac{1}{P}}}{2 P\left(\rho-\frac{1}{P}\right)^{\frac{5}{2}}}, \\
I_{3}=-\frac{\pi U\left(\frac{1}{2} ; 0 ; \frac{\eta^{2}}{4\left(\rho-\frac{1}{P}\right)}\right)}{\sqrt{\rho-\frac{1}{P}}}, \quad I_{4}=-\frac{\sqrt{2} \eta}{P \rho^{2}} G_{3,4}^{4,1}\left[\frac{\eta^{2}}{\rho} \mid \begin{array}{c}
-1 \\
\frac{-1}{2}, 0, \frac{1}{4}, \frac{3}{2}, 1
\end{array}\right], \quad I_{5}=\frac{\sqrt{\pi}}{2 \eta} G_{2,3}^{3,1}\left[\frac{\eta^{2}}{\rho} \mid \begin{array}{c}
1 \\
\frac{1}{2}, \frac{3}{2}, \frac{5}{2}
\end{array}\right] .
\end{gathered}
$$

normal random variable will obtain a value larger than $x$. Using integration by parts, we can show that

$$
P_{\text {appro }} \approx \frac{\beta M_{d_{\min }}}{4 \sqrt{\pi}} \int_{0}^{\infty} x^{-\frac{1}{2}} e^{-\frac{\beta^{2}}{4} x} F_{\gamma_{1}}(x) F_{\gamma_{2}}(x) d x .
$$

Thus, given the CDFs $F_{\gamma_{j}}(x)$, one can calculate the approximate BLER using (11). In the following two sub-sections, we consider the non-coherent and the coherent power coefficients, respectively.

\section{A. Non-Coherent Power Coefficient at Relays}

Assume that each relay node uses a non-coherent power coefficient given in (4). The CDF of $\gamma_{j}, F_{\gamma_{j}}(x)$, is rigorously derived in the following theorem.

Theorem 1: With non-coherent power coefficient, the CDF of $\gamma_{j}$ is

$$
\begin{aligned}
F_{\gamma_{j}}(x)= & 1-\eta_{j} \sqrt{x} e^{-\frac{x}{P}} K_{1}\left(\eta_{j} \sqrt{x}\right) \\
& +e^{-\frac{x}{P}} \int_{a_{j}-\frac{x}{P}}^{a_{j}} e^{-\left(y+\frac{\eta_{j}^{2} x}{4 y}\right)} d y
\end{aligned}
$$

where $a_{j}=\frac{Q_{j} x+\sqrt{Q_{j}^{2} x^{2}+4 P Q_{j}(1+2 P) x}}{2 P Q_{j}}, \eta_{j}=2 \sqrt{\frac{1+2 P}{P Q_{j}}}$, and $K_{1}(\cdot)$ is the modified first-order Bessel function of the second kind.

Proof: See the appendix.

We can further approximate the CDF in (12) for tractable BLER analysis using the mean value theorem. Let $\Lambda=$ $\left[a_{j}-\frac{x}{P}, a_{j}\right]$. With the mean value theorem, there exists a $\mu \in \Lambda$ such that

$$
\int_{a_{j}-\frac{x}{P}}^{a_{j}} e^{-\left(y+\frac{\eta_{j}^{2} x}{4 y}\right)} d y=\frac{x}{P} e^{-\left(\mu+\frac{\eta_{j}^{2} x}{4 \mu}\right)} .
$$

Let $z=\frac{(1+2 P) x}{P Q_{j}} \in \Lambda$. We have $|z-\mu| \leq x / P$. Since $x / P$ is small for large $P, F_{\gamma_{j}}(x)$ in (12) can be approximated, by replacing $\mu$ with $z$, as

$$
F_{\gamma_{j}}(x) \approx 1-\eta_{j} \sqrt{x} e^{-\frac{x}{P}} K_{1}\left(\eta_{j} \sqrt{x}\right)+\frac{x}{P} e^{-\left(\frac{x}{P}+\eta_{j} \sqrt{x}\right)} .
$$

For brevity, we assume that each relay node utilizes the same power, i.e., $Q_{1}=Q_{2}=Q .^{2}$ Thus, $\eta_{1}=\eta_{2}=2 \sqrt{\frac{1+2 P}{P Q}} \triangleq \eta$.

An approximate closed-form average BLER can be calculated using (11) and (13) to yield

$$
P_{\text {appro }} \approx \frac{M_{d_{\min }}}{2}+\frac{\beta M_{d_{\min }}}{4 \sqrt{\pi}} \sum_{t=1}^{5} I_{t}
$$

\footnotetext{
${ }^{2}$ The case of unequal relay power can be analyzed similarly.
}

where $I_{t}$ 's are given at the top of this page and $\rho=\left(\frac{2}{P}+\frac{\beta^{2}}{4}\right)$. Note that the first term, $\frac{M_{d_{\min }}}{2}$, in (14) is a constant. But it will not result in an error floor because its value will be canceled out by other terms. The closed-form expression includes $U(\cdot ; \cdot ; \cdot)$, the confluent hypergeometric function of the second kind, $\operatorname{Erfc}(\cdot)$, the complementary error function, and $G[\cdot]$, the Meijer's G-function [20], which are available in computer software packages such as MATLAB and MATHEMATICA. We omit the detailed derivations due to the space limit.

\section{B. Coherent Power Coefficient at Relays}

Assume that each relay has a coherent power coefficient given in (5). The single-relay selection in a two-relay twoway network with the coherent power coefficient has been extensively studied in [16].

With a rigorous analysis, the $\mathrm{CDF}$ for $F_{\gamma_{\hat{\imath}}}(x)$ is shown to be [16]

$$
\begin{aligned}
F_{\gamma_{j}}(x)=1 & -\frac{\kappa_{j}}{P} e^{-\left(1+2 \xi_{j}\right) \frac{x}{P}} K_{1}\left(\frac{\kappa_{j}}{P}\right) \\
& +e^{-\left(1+2 \xi_{j}\right) \frac{x}{P}} \int_{a_{j}-\left(1+\xi_{j}\right) \frac{x}{P}}^{a_{j}-\xi_{j} \frac{x}{P}} e^{-\left(y+\frac{\kappa_{j}^{2}}{4 P^{2} y}\right)} d y
\end{aligned}
$$

where $\xi_{j}=P / Q_{j}, \kappa_{j}=2 \sqrt{\xi_{j} x\left[1+\left(1+\xi_{j}\right) x\right]}$ and $a_{j}=$ $\frac{\left(\xi_{j}+\frac{1}{2}\right) x+\sqrt{\left(\xi_{j}+\frac{1}{2}\right)^{2} x^{2}+\xi_{j} x}}{P}$. Using the mean value theorem for large $P$, we have

$$
F_{\gamma_{j}}(x) \approx 1-\frac{\tilde{\kappa}_{j}}{P} x e^{-\frac{\theta_{j}}{P} x} K_{1}\left(\frac{\tilde{\kappa}_{j}}{P} x\right)+\frac{x}{P} e^{-\frac{\left(\theta_{j}+\tilde{\kappa}_{j}\right)}{P} x}
$$

where $\tilde{\kappa}_{j}=2 \sqrt{\xi_{j}\left(1+\xi_{j}\right)}$, and $\theta_{j}=1+2 \xi_{j}$. In [16], the CDF is further approximated using $K_{1}(x) \approx 1 / x$ by assuming that the transmit power of each node is high, i.e., $P, Q_{j} \gg 1$, to obtain

$$
F_{\gamma_{j}}(x) \approx 1-e^{-\frac{\theta_{j}}{P} x}+\frac{x}{P} e^{-\frac{\left(\theta_{j}+\kappa_{j}\right)}{P} x} .
$$

The approximate BLER is then derived using (11).

Since the approximation $K_{1}(x) \approx 1 / x$ is valid for large $x$ only, results in [16] are limited to high power constraint at each node. In this work, we revisit the same problem, and derive an approximate BLER using (16), which is valid for any power constraint. Again, assume that each relay has the same power, i.e., $Q_{1}=Q_{2}=Q$. Thus, $\xi_{1}=\xi_{2}=P / Q \triangleq \xi$, $\kappa_{1}=\kappa_{2}=2 \sqrt{\xi(1+\xi)} \triangleq \kappa$, and $\theta_{1}=\theta_{2}=1+2 \xi \triangleq \theta$. Using (11) and (16), an approximate average BLER expression 


$$
\begin{gathered}
J_{1}=\frac{\sqrt{\pi}}{P\left(\frac{\kappa}{P}+\frac{\theta}{P}+\frac{\beta^{2}}{4}\right)^{\frac{3}{2}}}, \quad J_{2}=\frac{3 \sqrt{\pi}}{4 P^{2}\left(\frac{2 \kappa}{P}+\frac{2 \theta}{P}+\frac{\beta^{2}}{4}\right)^{\frac{5}{2}}}, \\
J_{3}=-\frac{\sqrt{2 \pi \nu_{1}}}{\nu_{1}^{2}-\nu_{2}^{2}}\left(\left(\nu_{1}+\nu_{2}\right) K\left(\frac{\nu_{1}-\nu_{2}}{2 \nu_{1}}\right)-2 \nu_{2} E\left(\frac{\nu_{1}-\nu_{2}}{2 \nu_{1}}\right)\right) \quad \text { where } \nu_{1}=\frac{\kappa}{P} \text { and } \nu_{2}=\frac{\theta}{P}+\frac{\beta^{2}}{4}, \\
J_{4}=\frac{64 \sigma_{1} \Gamma\left(\frac{5}{4}\right)^{2} \Gamma\left(\frac{9}{4}\right)^{2}{ }_{3} F_{2}\left(\frac{1}{4}, \frac{5}{4}, \frac{9}{4} ; \frac{1}{2}, \frac{7}{4} ; \frac{\sigma_{2}^{2}}{4 \sigma_{1}^{2}}\right)-9 \sigma_{2} \Gamma\left(\frac{3}{4}\right)^{3} \Gamma\left(\frac{11}{4}\right)_{3} F_{2}\left(\frac{3}{4}, \frac{7}{4}, \frac{11}{4} ; \frac{3}{2}, \frac{9}{4} ; \frac{\sigma_{2}^{2}}{4 \sigma_{1}^{2}}\right)}{15 \sqrt{2 \pi} \sigma_{1}^{\frac{3}{2}}} \text { where } \sigma_{1}=\frac{\kappa}{P} \text { and } \sigma_{2}=\frac{2 \theta}{P}+\frac{\beta^{2}}{4}, \\
J_{5}=\frac{\sqrt{\frac{\pi \sigma_{1}}{2}}}{P\left(\left(\sigma_{1}+\sigma_{2}\right)^{2}-\sigma_{1}^{2}\right)^{2}}\left[\left(\left(\sigma_{1}+\sigma_{2}\right)^{2}+\sigma_{1}\left(4\left(\sigma_{1}+\sigma_{2}\right)+3 \sigma_{1}\right)\right) K\left(\frac{-\sigma_{2}}{2 \sigma_{1}}\right)-2\left(\left(\sigma_{1}+\sigma_{2}\right)^{2}+3 \sigma_{1}^{2}\right) E\left(\frac{-\sigma_{2}}{2 \sigma_{1}}\right)\right] .
\end{gathered}
$$

can be derived in closed-form as

$$
P_{\text {appro }} \approx \frac{M_{d_{\min }}}{2}+\frac{\beta M_{d_{\min }}}{4 \sqrt{\pi}} \sum_{t=1}^{5} J_{t}
$$

where $J_{t}$ 's are given at the top of this page. Here, $K(\cdot)$ and $E(\cdot)$ are the complete elliptic integral of the first and the second kind, respectively, and ${ }_{3} F_{2}(\cdot)$ is a generalized hypergeometric function [20]. They are available in computer software packages such as MATLAB and MATHEMATICA. We omit the detailed derivations due to the space limit.

\section{Single-Relay Selection in a Network with an ARBITRARY NUMBER OF RELAYS}

In this section, we consider a network with an arbitrary number of relays. Also, we consider the coherent power coefficient only for the following reasons. Firstly, simulation in Section VI shows that in two-way relay networks with two relays, the coherent power coefficient outperforms the non-coherent power coefficient. Secondly, for the two-way communications, the overhead cost to gain the instantaneous CSI $f_{j}$ and $g_{j}$ at $r_{j}$ is low. The relay can gain reliable estimations with only one pilot signal from each user and no feedback is needed. Finally, numerical results in Section VI also show that the approximation $K_{1}(x) \approx \frac{1}{x}$, while works well for the coherent power coefficient case, results in a loose CDF approximation for the non-coherent power coefficient case and is inappropriate for the BLER approximation.

Assume that all relays have the same power, i.e., $Q_{j}=Q$, for $j=1, \cdots, R$. Therefore, the CDF of $\gamma_{\hat{i}}$ can be calculated as $F_{\gamma_{\hat{\imath}}}(x)=\left[F_{\gamma_{j}}(x)\right]^{R}$. However, the BLER analysis is intractable with (16) because of the modified Bessel function. Instead, we use the approximation in (17). Thus, the BLER analysis in this selection is for the high transmit power regime. Its accuracy is justified by the simulation results in Section VI. This approximation also allows the diversity derivation. With (17), $F_{\gamma_{\hat{i}}}(x)$ can be approximated as

$$
F_{\gamma_{\hat{i}}}(x) \approx\left(1-e^{-\frac{\theta}{P} x}+\frac{x}{P} e^{-\frac{(\theta+\kappa)}{P} x}\right)^{R}, x \geq 0 .
$$

Using multinomial series expansion, (19) can be re-written as

$$
F_{\gamma_{\hat{i}}}(x) \approx \sum_{\substack{n_{1}, n_{2}, n_{3} \geq 0 \\ n_{1}+n_{2}+n_{3}=R}} \frac{R !}{n_{1} ! n_{2} ! n_{3} !} \frac{(-1)^{n_{2}}}{P^{n_{3}}} x^{n_{3}} e^{-\frac{\psi}{P} x}
$$

where $\psi=\theta\left(n_{2}+n_{3}\right)+\kappa n_{3}$.
Block Error Rate: With the aid of (11) and (20), the BLER can be evaluated as

$$
P_{\text {appro }} \approx \frac{M_{d_{\min }} \beta}{4 \sqrt{\pi}} \sum_{\substack{n_{1}, n_{2}, n_{3} \geq 0 \\ n_{1}+n_{2}+n_{3}=R}} \frac{R !(-1)^{n_{2}}}{n_{1} ! n_{2} ! n_{3} ! P^{n_{3}}} \frac{\Gamma\left(n_{3}-\frac{1}{2}\right)}{\left(\frac{\psi}{P}+\frac{\beta^{2}}{4}\right)^{n_{3}-\frac{1}{2}}}
$$

where $\Gamma(\cdot)$ is the Gamma function. Note that the BLER with $Q_{i} \neq Q_{j}(i \neq j)$ can be evaluated in the same way. However, it is difficult to give a general expression in a compact form.

Diversity Order: When $x \rightarrow 0$, we have $1-e^{-x} \approx x$. Therefore, for large $P$, (19) can be written as

$$
F_{\gamma_{\hat{i}}}(x) \approx \frac{x^{R}}{P^{R}}\left(\theta+e^{-\frac{\theta+\kappa}{P} x}\right)^{R} .
$$

Using the binomial expansion and (11), the BLER is approximated for large $P$ as

$P_{\text {appro }} \approx\left[\frac{M_{d_{M i n}} \beta}{4 \sqrt{\pi}} \sum_{m=0}^{R}\left(\begin{array}{c}R \\ m\end{array}\right) \frac{\theta^{R-m} \Gamma\left(R+\frac{1}{2}\right)}{\left(\frac{m(\theta+\kappa)}{P}+\frac{\beta^{2}}{4}\right)^{\left(R+\frac{1}{2}\right)}}\right] P^{-R}$

where $\left(\begin{array}{l}R \\ m\end{array}\right)=\frac{R !}{m !(R-m) !}$. The diversity order is thus $R$ which is the full diversity in a Rayleigh fading environment for a network with $R$ relays.

The relay selection algorithm can be performed at either end user, who knows all channels. The user can find the index of the relay with the highest worse end-to-end SNR and feedback its index. $\log R$ feedback bits are needed. Other strategies that require less or no feedback are available, but is out of the scope of this paper and will be explored later.

\section{Multiple-Relay Selection}

In this section, we generalize the opportunistic relaying scheme from single-relay selection to multiple-relay selection, in other words, more than one relays are allowed to participate in the second-phase of the communication. For one-way relay networks, multiple-relay selection is proposed in [6] and it is shown to have a much better performance than single-relay selection with a small amount of cost on the overhead.

Consider the two-way relay network in Fig. 1. When more than one relays are allowed to cooperate, for the relay signals to be added coherently at the two end users, during the second phase of communication, each relay should, in addition to amplifying the information, incorporate a phase shift to cancel 
the phases of its channels. The coefficient at the $i$ th relay should be $\alpha_{i} e^{j \varphi_{i}}$, where $\varphi_{i}=-\left(\angle f_{i}+\angle g_{i}\right)$, and $\angle \vartheta$ denotes the phase of a complex value $\vartheta$. Again, the coherent power coefficient in (5) is used. Assume that Relays $i_{1}, \cdots, i_{k}$ are chosen. Let $\mathcal{R}=\left\{i_{1}, \cdots, i_{k}\right\}$, which is the set of indices of the selected relays. Each end user receives a superposition of the relay transmissions as follows:

$$
\begin{aligned}
& y_{u_{1}}=\sum_{i \in \mathcal{R}} \alpha_{i}\left[\sqrt{P} f_{i}^{2} e^{j \varphi_{i}} s_{1}+\sqrt{P}\left|f_{i} g_{i}\right| s_{2}+f_{i} \tilde{v}_{i}\right]+w_{1} \\
& y_{u_{2}}=\sum_{i \in \mathcal{R}} \alpha_{i}\left[\sqrt{P} g_{i}^{2} e^{j \varphi_{i}} s_{2}+\sqrt{P}\left|f_{i} g_{i}\right| s_{1}+g_{i} \tilde{v}_{i}\right]+w_{2}
\end{aligned}
$$

where $\tilde{v}_{i}=v_{i} e^{j \varphi_{i}}$. After canceling the self-interference, $U_{1}$ and $U_{2}$ get:

$$
\begin{gathered}
\tilde{y}_{u_{1}}=\sqrt{P} \sum_{i \in \mathcal{R}} \alpha_{i}\left|f_{i} g_{i}\right| s_{2}+\sum_{i \in \mathcal{R}} \alpha_{i} f_{i} \tilde{v}_{i}+w_{1} \\
\tilde{y}_{u_{2}}=\sqrt{P} \sum_{i \in \mathcal{R}} \alpha_{i}\left|f_{i} g_{i}\right| s_{1}+\sum_{i \in \mathcal{R}} \alpha_{i} g_{i} \tilde{v}_{i}+w_{2} .
\end{gathered}
$$

The ML decoding rules of the two users are thus,

$$
\begin{aligned}
& U_{1}: \quad \hat{s}_{2}=\arg \min _{s \in \mathcal{S}}\left|\tilde{y}_{u_{1}}-\sqrt{P} \sum_{i \in \mathcal{R}} \alpha_{i}\right| f_{i} g_{i}|s| \\
& U_{2}: \quad \hat{s}_{1}=\arg \min _{s \in \mathcal{S}}\left|\tilde{y}_{u_{2}}-\sqrt{P} \sum_{i \in \mathcal{R}} \alpha_{i}\right| f_{i} g_{i}|s| .
\end{aligned}
$$

Note that the phase adjustment does not affect the statistical properties of the additive noise at the relay nodes, i.e., $v_{i}$ and $\tilde{v}_{i}$ have the same variance. Thus, the received SNRs at the two users when the relay subset $\mathcal{R}$ is selected are

$$
\begin{aligned}
& U_{1}: \quad \gamma_{u_{1}, \mathcal{R}}=\frac{P\left(\sum_{i \in \mathcal{R}} \alpha_{i}\left|f_{i} g_{i}\right|\right)^{2}}{1+\sum_{i \in \mathcal{R}} \alpha_{i}^{2}\left|f_{i}\right|^{2}} \\
& U_{2}: \quad \gamma_{u_{2}, \mathcal{R}}=\frac{P\left(\sum_{i \in \mathcal{R}} \alpha_{i}\left|f_{i} g_{i}\right|\right)^{2}}{1+\sum_{i \in \mathcal{R}} \alpha_{i}^{2}\left|g_{i}\right|^{2}}
\end{aligned}
$$

respectively. Based on our relay selection criterion explained in Section II, the multiple-relay selection scheme is thus to find the subset of $\{1,2, \cdots, R\}$ that results in the maximum worse end-to-end SNR. In other words,

$$
\mathcal{R}=\arg \max _{\mathcal{R} \subseteq\{1,2, \cdots, R\}} \min \left\{\gamma_{u_{1}, \mathcal{R}}, \gamma_{u_{2}, \mathcal{R}}\right\} .
$$

As there are $R$ relays and each relay has two choices, there are $2^{R}-1$ possibilities (the case that no relay cooperates is obviously not optimal). One can always find the optimal solution by exhaustive search. But the computational complexity is exponential in $R$, and the number of required feedback bits is $R$ since one bit for each relay is needed. For networks with large numbers of relays, having the amount of feedback bits linear in the network size is undesirable. Thus, the same as one-way relay networks, the real challenge of the problem is to find multiple-relay selection schemes with low complexity, good performance, and, at the same time, low feedback requirement. This motivates the relay ordering idea in one-way networks [6]. With a relay ordering, one can find the cooperating relays sequentially. Although it is proved that for one-way relay networks, the optimal relay ordering does not exist, a relay ordering function based on the end-to-end SNR is proposed which is shown to achieve the full diversity and near optimal performance with linear or quadratic complexity. In this paper, we generalize the idea to two-way networks and propose the worse end-to-end SNR as the relay ordering function. The proposed multiple-relay selection scheme is described in Algorithm 1.

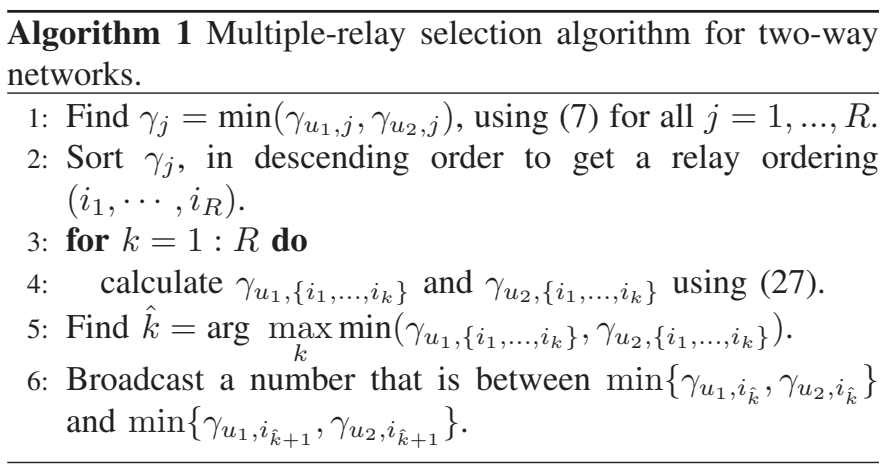

The relay selection scheme can be conducted by either end user who knows all the channels. Relay $j$ hears the value broadcasted by the end user and compares the value with its own worse end-to-end SNR, $\gamma_{j}$. If $\gamma_{j}$ is larger, Relay $j$ cooperates; otherwise, it does not cooperate. The scheme requires feedback of one positive number that is common to all relays. Thus, the required number of feedback bits is a fixed number whose value depends on the required precision. More efficient selection strategies may exist but is beyond the scope of this paper and will be explored later.

\section{Numerical and Simulation Results}

In this section, we give numerical and simulation results to verify our analysis, and to evaluate the performance of the relay selection schemes. QPSK is used as the modulation scheme in which $\beta=\sqrt{2}$ and $M_{d_{\min }}=2$.

Since the exact analytical expressions for the CDFs of the worse end-to-end SNRs make the BLER analysis intractable, two approximations have been introduced, using the mean value theorem in Section III (referred to as appro 1) and using $K_{1}(x) \approx \frac{1}{x}$ for large $x$ together with the mean value theorem in Section IV (referred to as appro 2). We justify the approximations by numerical results and simulation here. Fig. 2 compares the two approximations with its exact CDFs for both coherent and non-coherent cases when $P=Q=10$ $\mathrm{dB}$. The exact CDFs are generated by Monte-Carlo simulation. It shows that appro 1 is very close to the exact one. Similar phenomenon can be observed for other values of $P$. Therefore, the approximation for the single-relay selection in a two-relay network with coherent or non-coherent power coefficient in Section III is precise for a large range of $P$ and $Q$. However, appro 2 is not as close to the exact one as appro 1, and the 


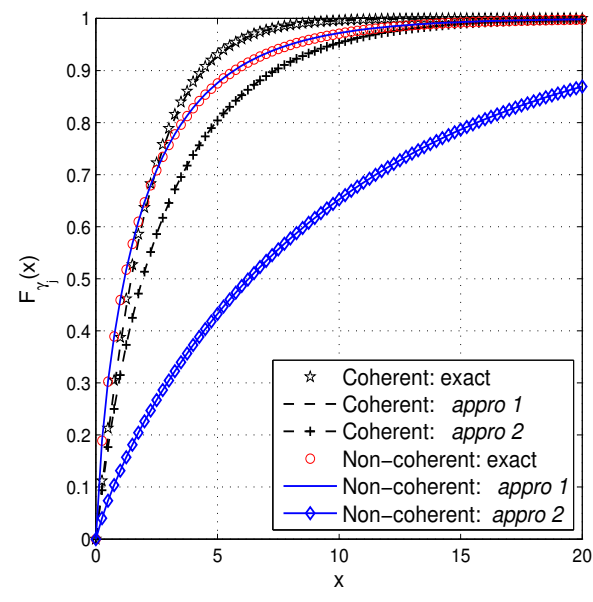

Fig. 2. Comparison of CDFs.

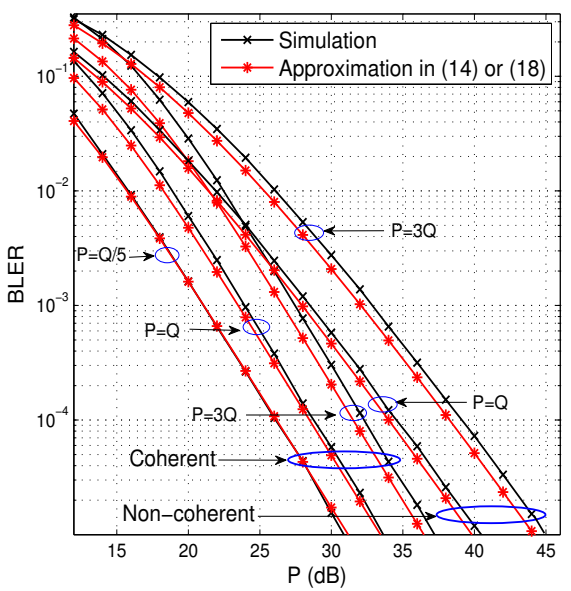

Fig. 3. Single-relay selection with coherent and non-coherent power coefficients.

gap from the exact $\mathrm{CDF}$ is much larger in the non-coherent case than in the coherent case.

In Fig. 3, the BLERs of a two-way network with two relays under the proposed single-relay selection scheme are shown. The non-coherent power coefficient case is shown under two power scenarios: 1) $P=Q$ and 2) $P=3 Q$. The coherent power coefficient case is shown under three power scenarios: 1) $P=Q / 5,2) P=Q$, and 3) $P=3 Q$. For all cases, networks with coherent power coefficient outperform those with non-coherent power coefficient. To achieve $10^{-5}$ BLER, the coherent case is approximately $5 \mathrm{~dB}$ and $6 \mathrm{~dB}$ better when $P=Q$ and $P=3 Q$, respectively. With coherent power coefficient, the difference between $P=Q$ and $P=3 Q$ to achieve $10^{-5}$ BLER is $3.5 \mathrm{~dB}$, while with non-coherent power coefficient, the difference is $5 \mathrm{~dB}$. This means the coherent power coefficient case is less sensitive to the relay power fluctuation. The figure also shows that the analytical approximation is a tight lower bound on the BLER. For all

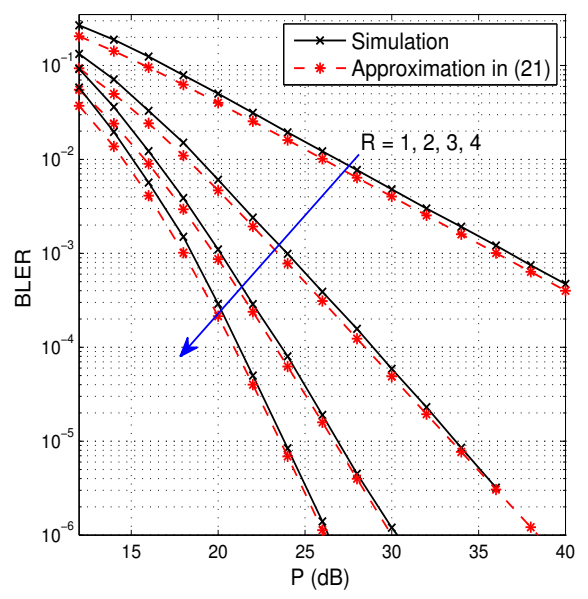

Fig. 4. Single-relay selection with coherent power coefficient.

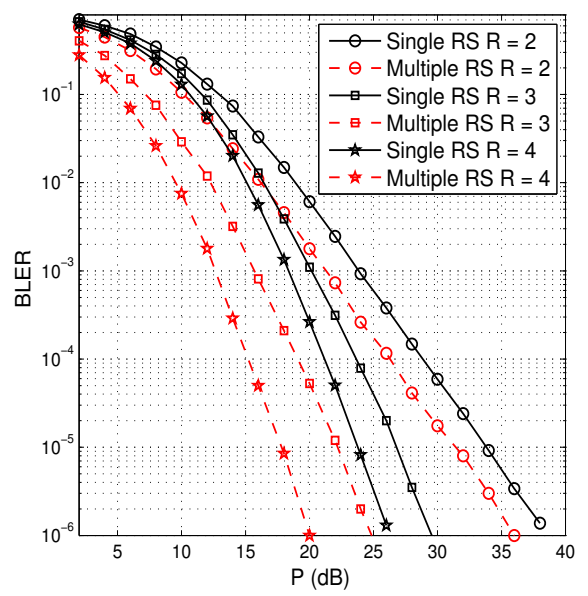

Fig. 5. Multiple-relay selection with coherent power coefficient.

network scenarios, it approximates the BLER with less than $1 / 2 \mathrm{~dB}$ difference.

In Fig. 4, we consider relay networks with $R=1,2,3,4$ for $P=Q$ to see the diversity result. It shows clearly that the diversity gain changes from 1 to 4 when $R$ varies from 1 to 4. This is consistent with our result that diversity $R$ can be achieved. Further, the BLER approximation in (21) acts as a lower bound which is consistent with our discussion before, and it is a tight bound for large $P$. For each case of $R$, the gap between the simulation curve and the approximation curve is less than $0.3 \mathrm{~dB}$ at $10^{-6}$ BLER.

Fig. 5 shows BLER performance of multiple-relay selection scheme for $R=2,3$, and 4, where 'RS' stands for 'relay selection'. For comparison, performance of single-relay selection is also given in Fig. 5. It shows that both schemes have the same diversity order $R$, but multiple-relay selection has a larger array gain. The array gain increases as the number of relays increases. To achieve $10^{-6}$ BLER, multiple-relay selection outperforms the single-relay selection by approximately $2 \mathrm{~dB}$, 
$5 \mathrm{~dB}$, and $7 \mathrm{~dB}$ for $R=2, R=3$, and $R=4$, respectively.

\section{CONCLUSION}

A two-way relay network with multiple AF relays is considered for both non-coherent and coherent power coefficients at the relays. A simple relay selection scheme which chooses the relay that results in the highest worse receive SNR of the two users is proposed. Then, a multiple-relay selection scheme is considered by ordering the set of relays in descending order of the worse receive SNR of the two users. The BLER approximation for single-relay selection in a two-relay network is shown to be accurate for any transmit power. The approximation used in single-relay selection with an arbitrary number of relays is shown to be accurate when the transmit power is high. It is also shown that a two-way relay network with coherent power coefficient outperforms that with noncoherent power coefficient. Both the single-relay selection and the multiple-relay selection schemes achieve full diversity, while the latter achieves a larger array gain than the former.

\section{APPENDIX}

Let $\alpha=\left|f_{j}\right|^{2}$ and $\zeta=\left|g_{j}\right|^{2}$, which have exponential distributions. For any $x>0$, we define $b_{j} \triangleq \frac{x}{P}$ and $a_{j} \triangleq$ $\frac{Q_{j} x+\sqrt{Q_{j}^{2} x^{2}+4 P Q_{j}(1+2 P) x}}{2 P Q_{j}}$. For $x \geq 0$, we have $0 \leq b_{j} \leq a_{j}$. Therefore, the probability of $\gamma_{j} \geq x$ can be given as

$$
\begin{aligned}
\mathbb{P}\left(\gamma_{j} \geq x\right) & =\int_{0}^{b_{j}} \mathbb{P}\left(\gamma_{j} \geq x \mid t=\alpha\right) e^{-t} d t \\
& +\int_{b_{j}}^{a_{j}} \mathbb{P}\left(\gamma_{j} \geq x \mid t=\alpha\right) e^{-t} d t \\
& +\int_{a_{j}}^{\infty} \mathbb{P}\left(\gamma_{j} \geq x \mid t=\alpha\right) e^{-t} d t .
\end{aligned}
$$

When $\alpha \in\left[0, b_{j}\right)$, we have

$$
\begin{aligned}
& (P \alpha-x)<0<\frac{x(1+2 P)}{\zeta Q_{j}} \Rightarrow \frac{P Q_{j} \alpha \zeta}{1+2 p+\zeta Q_{j}}<x \\
& \Rightarrow \gamma_{u_{1}, j}<x \Rightarrow \gamma_{j}<x .
\end{aligned}
$$

Thus, the first integral in (28) is zero. When $0 \leq b_{j} \leq \alpha$,

$$
\begin{aligned}
\gamma_{u_{1}, j} \geq x & \Leftrightarrow P Q_{j} \alpha \zeta \geq\left(Q_{j} \alpha+2 P+1\right) x \\
& \Leftrightarrow \zeta \geq \frac{Q_{j} \alpha+(1+2 P)}{P Q_{j} \alpha} x \triangleq c_{1, j} .
\end{aligned}
$$

Similarly, $\gamma_{u 2, j} \geq x \Leftrightarrow \zeta \geq \frac{1+2 P}{P Q_{j} \alpha-Q_{j} x} x \triangleq c_{2, j}$. Therefore,

$$
\begin{aligned}
& \gamma_{j} \geq x \Leftrightarrow \gamma_{u_{1}, j}>x, \\
& \gamma_{u_{2}, j}>x \Leftrightarrow \zeta \geq \max \left\{c_{1, j}, c_{2, j}\right\}, \\
& c_{1, j} \gtreqless c_{2, j} \Leftrightarrow P Q_{j} \alpha^{2}-Q_{j} x \alpha-x(1+2 P) \gtreqless 0, \\
& P Q_{j} \alpha^{2}-Q_{j} x \alpha-x(1+2 P)=0 \Leftrightarrow \alpha=a_{j} .
\end{aligned}
$$

Since $a_{j} \geq b_{j}$, we can conclude that for $\alpha \geq b_{j}$,

$$
c_{1, j} \gtreqless c_{2, j} \Leftrightarrow \alpha \gtreqless a_{j} .
$$

Therefore, (28) can be re-written as

$$
\mathbb{P}\left(\gamma_{j} \geq x\right)=\int_{b_{j}}^{a_{j}} e^{-c_{2, j}} e^{-t} d t+\int_{a_{j}}^{\infty} e^{-c_{1, j}} e^{-t} d t .
$$

Using the definitions of $c_{1, j}$ and $c_{2, j}$ given above, (29) can be evaluated with the aid of the transformation $t-\frac{x}{P}=y$ and [20, eq. 3.324.1], to yield

$$
\begin{aligned}
\mathbb{P}\left(\gamma_{j} \geq x\right)= & \eta_{j} \sqrt{x} e^{-\frac{x}{P}} K_{1}\left(\eta_{j} \sqrt{x}\right) \\
& -e^{-\frac{x}{P}} \int_{a_{j}-\frac{x}{P}}^{a_{j}} e^{-\left(y+\frac{\eta_{j}^{2} x}{4 y}\right)} d y
\end{aligned}
$$

where $\eta_{j}=2 \sqrt{\frac{(1+2 P)}{P Q_{j}}}$. The CDF of $\gamma_{j}$ can be written as $F_{\gamma_{j}}(x)=\mathbb{P}\left(\gamma_{j} \leq x\right)=1-\mathbb{P}\left(\gamma_{j} \geq x\right)$, which proves (12).

\section{REFERENCES}

[1] A. Sendonaris, E. Erkip, and B. Aazhang, "User cooperation diversitypart I: System description," IEEE Trans. Commun., vol. 51, no. 11, pp. 1927-1938, Nov. 2003.

[2] A. Sendonaris, E. Erkip, and B. Aazhang, "User cooperation diversitypart II: Implementation aspects and performance analysis," IEEE Trans. Commun., vol. 51, no. 11, pp. 1939-1948, Nov. 2003.

[3] Y. Jing and B. Hassibi, "Distributed space-time coding in wireless relay networks," IEEE Trans. Wireless Commun., vol. 5, no. 12, pp. 3524 3536, Dec. 2006.

[4] M. O. Hasna and M. S. Alouini, "End-to-end performance of transmission systems with relays over Rayleigh-fading channels," IEEE Trans. Wireless Commun., vol. 2, no. 6, pp. 1126-1131, Nov. 2003.

[5] M. Hasna and M.-S. Alouini, "Harmonic mean and end-to-end performance of transmission systems with relays," IEEE Trans. Commun., vol. 52, no. 1, pp. 130 - 135, Jan. 2004.

[6] Y. Jing and H. Jafarkhani, "Single and multiple relay selection schemes and their achievable diversity orders," IEEE Trans. Wireless Commun., vol. 8, no. 3, pp. $1414-1423$, Mar. 2009.

[7] E. Beres and R. Adve, "Selection cooperation in multi-source cooperative networks," IEEE Trans. Wireless Commun., vol. 7, no. 1, pp. 118 -127 , Jan. 2008.

[8] C. E. Shannon, "Two-way communication channels," in Proc. 4th Berkeley Symp. Math. Stat. Prob., 1961, pp. 611-644.

[9] B. Rankov and A. Wittneben, "Spectral efficient signaling for halfduplex relay channels," in Proc. the Thirty-Ninth Asilomar Conf. on Signals, Systems and Computers, Nov. 2005, pp. 1066 - 1071.

[10] S. J. Kim, P. Mitran, and V. Tarokh, "Performance bounds for bidirectional coded cooperation protocols," IEEE Trans. Inform. Theory, vol. 54, no. 11, pp. 5235-5241, Nov. 2008.

[11] T. Oechtering and H. Boche, "Bidirectional regenerative half-duplex relaying using relay selection," IEEE Trans. Wireless Commun., vol. 7 , no. 5, pp. $1879-1888$, May 2008.

[12] L. Ding, M. Tao, F. Yang, and W. Zhang, "Joint scheduling and relay selection in one- and two-way relay networks with buffering," in Proc. IEEE Int. Conf. Commun. (ICC'09), June 2009.

[13] K.-S. Hwang, Y.-C. Ko, and M.-S. Alouini, "Performance bounds for two-way amplify-and-forward relaying based on relay path selection," in Proc. IEEE Veh. Technol. Conf. (VTC Spring'09), Apr. 2009.

[14] L. Song, G. Hong, B. Jiao, and M. Debbah, "Joint relay selection and analog network coding using differential modulation in two-way relay channels," IEEE Trans. Veh. Technol., 2010, accepted.

[15] M. Ju and I.-M. Kim, "Joint relay selection and opportunistic source selection in bidirectional cooperative diversity networks," IEEE Trans. Veh. Technol., 2010, accepted.

[16] Y. Jing, "A relay selection scheme for two-way amplify-and-forward relay networks," in Proc. Int. Conf. on Wireless Commun. Signal Processing (WCSP 2009), Nov. 2009.

[17] J. N. Laneman, D. N. C. Tse, and G. W. Wornell, "Cooperative diversity in wireless networks: Efficient protocols and outage behavior," IEEE Trans. Inform. Theory, vol. 50, no. 12, pp. 3062-3080, Dec. 2004.

[18] C. Patel, G. Stuber, and T. Pratt, "Statistical properties of amplify and forward relay fading channels," IEEE Trans. Veh. Technol., vol. 55, no. 1, pp. 1-9, Jan. 2006.

[19] S. Atapattu, N. Rajatheva, and C. Tellambura, "Performance analysis of TDMA relay protocols over Nakagami- $m$ fading," IEEE Trans. Veh. Technol., vol. 59, no. 1, pp. 93-104, Jan. 2010.

[20] I. S. Gradshteyn and I. M. Ryzhik, Table of Integrals, Series, and Products, 6th ed. Academic Press, Inc., 2000. 